\title{
Uma ajuda na análise e interpretação de informação da aptidão física de crianças e jovens provenientes de amostras de grande dimensão. Um tutorial centrado na modelação hierárquica ou multinível
}

\author{
José A.R. Maia ${ }^{1}$ \\ Rui Garganta 1 \\ André Seabra 1 \\ Vítor Lopes 2 \\ Simonete Silva 1 \\ Alcibíades Bustamante 1 \\ Rogério César Fermino ${ }^{1}$ \\ Duarte Freitas 3 \\ António Prista 4 \\ Cássio Meira Jr ${ }^{5}$
}

https://doi.org/10.5628/rpcd.07.03.379

\section{RESUMO}

Este estudo pretende apresentar, de modo didáctico, a utilização da modelação hierárquica ou multinível na análise e interpretação do desempenho motor de crianças e jovens a partir da prova da corrida-marcha da milha. As diferentes etapas da modelação são mencionadas, analisando o comportamento dos resultados a partir dos output's do software utilizado, o HLM 6.02. Cada etapa da análise é devidamente esclarecida e mencionada a sua relevância em termos interpretativos dos resultados.

Palavras-chave: modelação, hierarquia, desempenho motor, crianças
1 Faculdade de Desporto, Universidade do Porto, Portugal

2 Escola Superior de Educação

Instituto Politécnico de Bragança, Portugal

$3 \mathrm{U}$ niversidade da $\mathrm{M}$ adeira, Portugal

4 Faculdade de Ciências de Educação Física e D esporto

U niversidade Pedagógica de M oçambique, M oçambique

5 Escola de Educação Física e Esporte

Universidade de São Paulo - Brasil
ABSTRACT

Helping in analyzing and interpreting information from physical fitness of children gathered in large samples. A tutorial based on hierarchical or multilevel modelling

The aim of this study is to present, didactically, the use of hierarchical or multilevel modelling in analysing and interpreting children's motor performance, namely the one mile run-walk. We mention briefly the steps of modelling, analysing the results from the output of the software used, HLM 6.02. Each step is duly explained, and presented its relevance in interpretative terms.

Key-words: M odelling, hierarchy, motor performance, children 


\section{INTRODUÇÃO}

Os estudos de natureza epidemiológica acerca do estado, ou nível de aptidão física (AptF) de crianças e jovens lidam, necessariamente, com amostras de grandes dimensões - normalmente na casa das centenas ou milhares. Exemplos bem ilustrativos deste tipo de pesquisas em Portugal são os de Almeida et al.(1) em 768 crianças e jovens dos 10 aos 16 anos, de mais de 30 escolas do Concel ho de Lamego, de Ferreira et al.(9) em 720 jovens de ambos os sexos dos 10 aos 18 anos do Concelho de Viseu provenientes de 33 escolas, de Freitas et al.(10) em 1470 observações do seu estudo na região autónoma da Madeira oriundas de 29 escolas e de Maia et al.(17) em 3744 crianças dos seis aos 10 anos de idade de 57 escolas provenientes de oito Ilhas do arquipélago dos Açores. No Brasil, os exemplos são também cada vez mai ores, de que destacamos os trabalhos de Matsudo (19), Nahas et al.(20), Guedes e Guedes(13), Marcondes et al.(18), Waltrick e Duarte(29).

Estes estudos são de natureza transversal, observacionais na sua essência, cujos propósitos se podem situar em vários planos de que salientamos os seguintes: (1) descrever o nível ou estado de aptidão física relacionada á saúde (AptFS) das crianças e jovens em função do sexo e idade, com recurso a medidas descritivas bem conhecidas (média, desvio-padrão, mínimo e máximo), e raras vezes interval os de confiança (IC) para as respectivas médias; (2) avaliar aspectos do dimorfismo sexual, em que estas diferenças são atribuídas a um conjunto variado de factores (peso, altura, índice de massa corporal - IMC, valores de actividade física - ActF, estatuto sócio-económico, espaços habitacionais, etc.); (3) apresentar cartas centílicas do comportamento dos valores das diferentes provas de aptidão, propondo valores de referência que podem ser da maior importância em termos educativos, pedagógicos e de saúde pública.

Raras vezes encontramos esforços de model ação do desempenho das crianças e jovens a partir de um qualquer posicionamento biológico e/ ou cultural, ou orientado por uma teoria do desempenho motor (se é que tal teoria existe, não obstante os relevantes esforços de N evill e Holder(21). Para além deste facto que consideramos indesmentível, um dos principais problemas do tipo de pesquisa anteriormente referenciado reside na circunstância de dirigir toda a atenção para o nível informacional mais baixo de uma vasta hierarquia, ou seja, os sujeitos. Ora estes estudos, de larga escala, real izam-se em grandes espaços territoriais, amostrando crianças de diferentes escolas, que estão localizadas em regiões distintas do ponto de vista socio-económico. Crianças, escolas, espaços de local ização geográfica expressam uma estrutura hierárquica de forte dependência relacional que exige uma abordagem mais ecológi$\mathrm{ca}(8,15,25)$, que não ignore os problemas da heterogeneidade das rectas de regressão, dependência das observações e agregação(12,14, 24, 30).

Como bem demonstraram diferentes pesquisas em contexto escolar( $(4,11,23,26,28)$, é impossível pensar que o desempenho de crianças e jovens seja independente da dimensão das turmas, da qualidade diversificada dos professores, das condições distintas das escolas, e por aí adiante.

Estamos pois na presença de informação multinível ou hierárquica que reclama uma atenção urgente sob pena de se perder uma parte substancial da sua qualidade interpretativa, bem como se incorre no risco de se concluir de modo reducionista sobre o desempenho motor de crianças e jovens. Já chamamos a atenção para este facto no espaço da língua portuguesa(16) e os exemplos de outros países são bem claros nos domínios da performance motora de bebés(6), desenvolvimento do consumo máximo de $\mathrm{O}_{2}(2,3)$, e clima motivacional nas aulas de educação física (EF)(22). Face à reduzida expressão de textos di dácticos acerca do uso da model ação hierárquica ou multinível ( $M H M N$ ) quando a estrutura dos dados é de natureza eminentemente transversal, é nosso propósito apresentar um documento que auxilie os leitores no seu uso mais extenso, e sobretudo na necessidade de um maior cuidado no delineamento das suas pesquisas, ousadia no lançamento das hipóteses e maior robustez e elegância na análise e interpretação dos resultados.

Deste modo este trabalho está dividido em três partes: na primeira apresentamos o problema com base em informação concreta de um estudo acerca da AptF de crianças e jovens. A segunda refere-se aos aspectos da MHMN de um modo didáctico e sequencial a partir do lançamento de um conjunto diversificado de questões que colocaremos aos dados. $\mathrm{Na}$ terceira, apresentaremos partes do output do progra- 


\begin{tabular}{|c|c|c|c|c|c|c|}
\hline & & & & & & \\
\hline LEVEL-1 DESCRIPTIVE & STATI & CS (rel & as aos & 10s) & & descritivas dos dois pla- \\
\hline VARIABLE NAME & $\mathrm{N}$ & MEAN & SD & MINIMUM & MAXIMUM & $6.02)$ \\
\hline IDADE & 1779 & 7.69 & 1.30 & 6.00 & 10.00 & \\
\hline SEXO & 1779 & 0.52 & 0.50 & 0.00 & 1.00 & \\
\hline ACTF & 1779 & 77.79 & 27.57 & 11.00 & 161.00 & \\
\hline IMC & 1779 & 16.81 & 2.54 & 10.36 & 39.05 & $\begin{array}{l}\text { *Infra-estruturas escola- } \\
\text { res e recursos humanos }\end{array}$ \\
\hline LEVEL-2 DESCRIPTIVE & STATI & CS (rel & as ao & escolar & & $\begin{array}{l}\text { "mais relevantes" por } \\
\text { agrupamento escolar } \\
\text { (Meio: } 0=\text { =tipicamente }\end{array}$ \\
\hline VARIABLE NAME & $\mathrm{N}$ & MEAN & SD & MINIMUM & MAXIMUM & $\begin{array}{l}\text { rural; } 1=\text { rural; } 2=\text { =urbano. } \\
\text { Recreio: } 0=\text { =não tem; }\end{array}$ \\
\hline MEIO & 53 & 0.77 & 0.67 & 0.00 & 2.00 & $1=e s p a c ̧ o$ pequeno, mas \\
\hline VEST & 53 & 0.04 & 0.19 & 0.00 & 1.00 & não permite a prática de \\
\hline RECREIO & 53 & 1.79 & 0.63 & 0.00 & 3.00 & $\begin{array}{l}\text { qualquer des porto; } \\
2=e s p a c o \text { que permite a }\end{array}$ \\
\hline GINAS & 53 & 0.04 & 0.19 & 0.00 & 1.00 & prática de desporto; \\
\hline MATE & 53 & 0.02 & 0.14 & 0.00 & 1.00 & 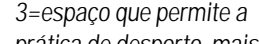 \\
\hline PROF & 53 & 0.02 & 0.14 & 0.00 & 1.00 & $\begin{array}{l}\text { pratica de desporto, mais } \\
\text { um ou dois campos de }\end{array}$ \\
\hline NATA & 53 & 0.72 & 0.45 & 0.00 & 1.00 & futebol de cinco. Demais \\
\hline EFISICA & 53 & 0.08 & 0.27 & 0.00 & 1.00 & $\begin{array}{l}\text { indicadores: } 0=\text { não e } \\
1=\text { sim) }\end{array}$ \\
\hline
\end{tabular}

ma que utilizaremos, o HLM 6.02 colando-as ao texto e interpretando o seu significado.

No sentido de não repetirmos informação previamente referenciada, solicitamos a todos os interessados nesta abordagem a leitura da parte final do texto de Sousa e Maia(27) concretamente o ponto sete dessa obra, onde o leitor encontrará um breve roteiro auxiliador de pesquisadores que pretendam utilizar este tipo de metodologia nos seus estudos. Chamamos a atenção do leitor que não é nosso propósito abordar a complicada estrutura estatística formal e aspectos computacionais da MHMN [ sobre estas matérias consultar Raudenbush e Bryk(24), Goldstein et al.(12) ], mas tão somente apresentar de uma forma didáctica as etapas sequenciais da análise, pensando sempre na óptica do utilizador deste tipo de metodologia e procedimento de análise [ ver o texto editado por Corgeau (7) acerca de aspectos filosóficos, metodológicos e analíticos da MHMN]. Não obstante lidarmos exclusivamente com o software HLM 6.0 (a versão estudante é gratuita bem como o respectivo manual, o site oficial possui exemplos comentados muito interessantes e altamente didácticos; é um dos softwares de MHMN mais “amigável" para os utilizadores), isto não significa que seja o único disponível, ou o melhor (sobre esta matéria consultar refa 16 ).

Os dados para análise

O Concelho de Amarante pertence ao distrito do Porto, fica situado na região norte do país, tendo 40 freguesias distribuídas por uma área de $301,5 \mathrm{Km} 2$. Este Concelho é atravessado por zonas urbanas e litorais com forte variabilidade económica. A sua rede escolar está distribuída por seis agrupamentos, com um total de 73 escolas do primeiro ciclo do ensino básico. Foram amostradas 92\% $(n=2940)$ das crianças dos seis aos 14 anos do universo escolar. Contudo, dado que o efectivo a partir dos 10 anos de idade era reduzido, consideramos somente 2801 crianças. A inspecção detalhada dos dados obrigou à consideração, para efeitos de apresentação da MHMN, de somente 1779 indivíduos distribuídos por 53 escolas dos seis agrupamentos (Quadro 1). É evidente que a frequência de alunos por escola é bastante diversificada dadas as características demografias e orográficas de cada freguesia.

A AptFS foi avaliada de acordo com a bateria ameri- 
Quadro 2

Resultados do

modelo nulo

(partes do out-

put do HLM

$6.02)$.

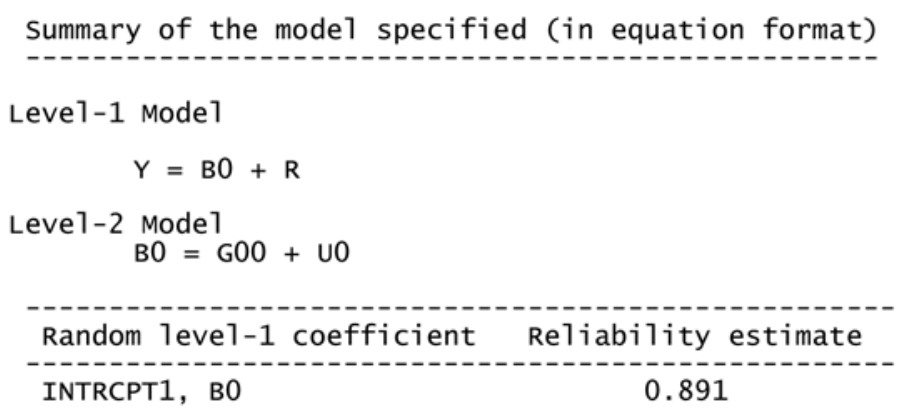

-

Final estimation of fixed effects:

\begin{tabular}{|c|c|c|c|c|c|}
\hline Fixed Effect & Coefficient & $\begin{array}{l}\text { Standard } \\
\text { Error }\end{array}$ & T-ratio & $\begin{array}{l}\text { Approx. } \\
\text { d.f. }\end{array}$ & P-value \\
\hline $\begin{array}{l}\text { INTRCPT1, B0 } \\
\text { INTRCPT } 2, \text { G00 }\end{array}$ & 11.715144 & 0.212180 & 55.213 & 52 & 0.000 \\
\hline
\end{tabular}

Final estimation of variance components:

\begin{tabular}{|c|c|c|c|c|c|}
\hline Random Effect & $\begin{array}{l}\text { Standard } \\
\text { Deviation }\end{array}$ & $\begin{array}{l}\text { Variance } \\
\text { Component }\end{array}$ & $d f$ & Chi-square & P-value \\
\hline $\begin{array}{l}\text { INTRCPT1, } \\
\text { Teve1-1, }\end{array}$ & $\begin{array}{l}1.45808 \\
2.17870\end{array}$ & $\begin{array}{l}2.12600 \\
4.74675\end{array}$ & 52 & 796.96965 & 0.000 \\
\hline
\end{tabular}

Statistics for current covariance components model

$\begin{aligned} & \text { Deviance }=7948.941405 \\ & \text { Number of estimated parameters }=3\end{aligned}$

cana Fitnessgram e a ActF a partir do questionário de Godin e Shephard(11). A determinação de aspectos relativos às condições sócio-económicas de implantação da escola, das características das infra-estruturas disponíveis, bem como do material humano disponível para leccionar aulas de EF para as crianças estão extensamente detal hadas no livro de Sousa e Maia(27).

Para evitar a construção de um texto muito extenso, iremos centrar a nossa atenção, exclusi vamente, na prova da milha. Chamamos a atenção dos leitores que foram utilizadas diferentes estratégias e procedimentos de análise (bivariada e multivariada) para avaliar a qualidade de toda a informação recol hida, e cujos resultados são altamente satisfatórios e indiciadores da elevada qualidade dos dados disponíveis. Pode parecer descabido e incorrecto o cál culo de médias com variáveis binárias tal como é ilustrado no output anterior. Contudo, não deixa de ser esclarecedor tal cálculo. Por exemplo, se tivéssemos o mesmo número de meninos e meninas, a média seria 0,50. Do mesmo modo, se pode entender que o número de escolas que têm ginásios (GINAS) é extremamente baixa, praticamente insignificante, dado que a média é 0,04 . Deixamos ao leitor a interpretação das outras médias.

\section{ETAPAS NA ANÁLISE DA MHMN}

Etapa no1: ANOVA de efeitos aleatórios (do inglês random effects ANOVA)

A primeira etapa de modelação consiste em determinar a extensão da variação que existe ao nível dos alunos e das escolas, isto é, estimar a magnitude da variância nos dois níveis da hierarquia informacio- 
nal. Este primeiro esforço é solucionado com base nos resultados da ANOVA de efeitos al eatórios (também designado de modelo nulo, ou intercept only model, dado que não contém qualquer variável que explique, em qualquer dos níveis da hierarquia, a variação encontrada). Os resultados estão no Quadro 2 , que passaremos a explicar com base no seguinte conjunto de questões (ver Quadro 2).

Será possível ter uma ideia da grande média do desempenho na prova da milha de todos os alunos de todas as escolas, algo semelhante a uma grande média? A grande média na corrida marcha da milha é de 11,72 minutos. Com $95 \%$ de confiança $\left({ } C_{95 \%}\right)$, a média do desempenho nesta prova que pretende marcar a aptidão cárdio-respiratória de todas as crianças dos seis aos 10 anos de idade do Concelho de Amarante, situa-se entre 11,31 e 12,13 minutos $[11,72 \pm 1,96(0,21)]$.

Qual é a extensão da variância entre alunos e entre escolas? A variância inter-individual dos alunos de todas as classes e de ambos os sexos (efeito ao nível dos alunos) é de 4,75 ( $p<0,001$ ); a variância que marca as diferenças de desempenho na milha é de $2,13$ ( $p<0,001)$ ao nível das escolas.

Será possível ter uma ideia da magnitude da dependência do desempenho à circunstância dos alunos pertencerem a diferentes escolas? Isto é, qual a magnitude do efeito das escolas? A presença de uma estrutura hierárquica nos resultados requer o cál culo do coeficiente de correlação intraclasse (rho), cuja fórmula é $2,12600 /(2,12600+4,74675) \approx 0,31$. 31\% da variância total do desempenho é devido a um efeito da escola, o que é um valor substancial. Este é, de facto, o passo essencial para justificar a presença da modelação hierárquica. Por exemplo, no estudo dos Açores(17), o valor da correlação intraclasse para a mesma prova foi de $7,5 \%$, na pesquisa de Zhu(30) no mesmo intervalo de idade foi de $22,4 \%$, e em Maia et al.(16) foi de $22 \%$, mas em crianças e jovens dos 10 aos 18 anos de idade. Estes valores atestam, de uma forma substancial, a estrutura multinível do desempenho.

Qual é a magnitude da fiabilidade da média do desempenho de cada escola? O seu valor é extremamente elevado, sendo de 0,891 . Este valor salienta a el evada qualidade dos resultados em termos da sua fiabilidade.
Será possível testar a hipótese das escolas terem a mesma média de desempenho na prova da milha? Claro que sim. Se tivessem a mesma média, a componente de variância na parte aleatória do modelo (random part no output relativo ao intercept) seria não significativa, o que não se passa. $O$ interval o de confiança a 95\% para a média das escolas é de 8,86 a $14,58\left[11,72 \pm 1,96(2,13)^{1 / 2}\right]$, o que significa uma amplitude substancial dos níveis de aptidão cárdiorespiratória entre escolas. Há escolas com um bom desempenho, e outras com um desempenho de menor qualidade (Figura 1).

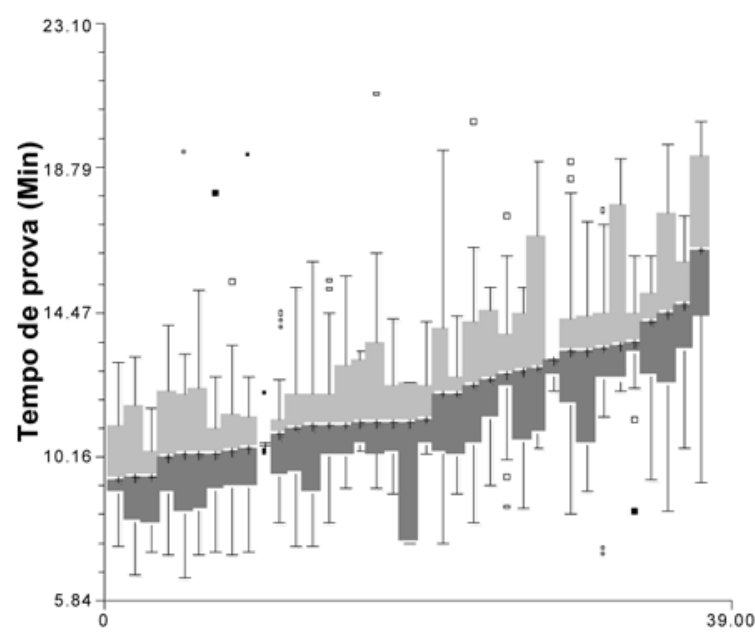

Figura 1. Amplitude do desempenho (diagrama de extremos e quartis) de uma amostra aleatória ordenada ( $70 \%$ ) das escolas em função da média do seu desempenho. Na abcissa encontram-se escolas, e na ordenada o desempenho expresso em minutos ( gráfico obtido no HLM 6.02).

Etapa no2: Modelo de efeitos fixos (do inglês Level-1 random intercept model)

A segunda etapa implica um esforço de modelação do desempenho a partir dos predictores disponíveis ao nível dos alunos, isto é, no primeiro nível da hierarquia informacional: idade, sexo, níveis de ActF e o IMC. O problema a ser esclarecido é saber se o desempenho melhora com a idade, se os meninos percorrem a distância num tempo menor, se os mais activos têm performances melhores, e se os que têm valores de IMC mais elevados são penalizados no seu desempenho (Quadro 3). 


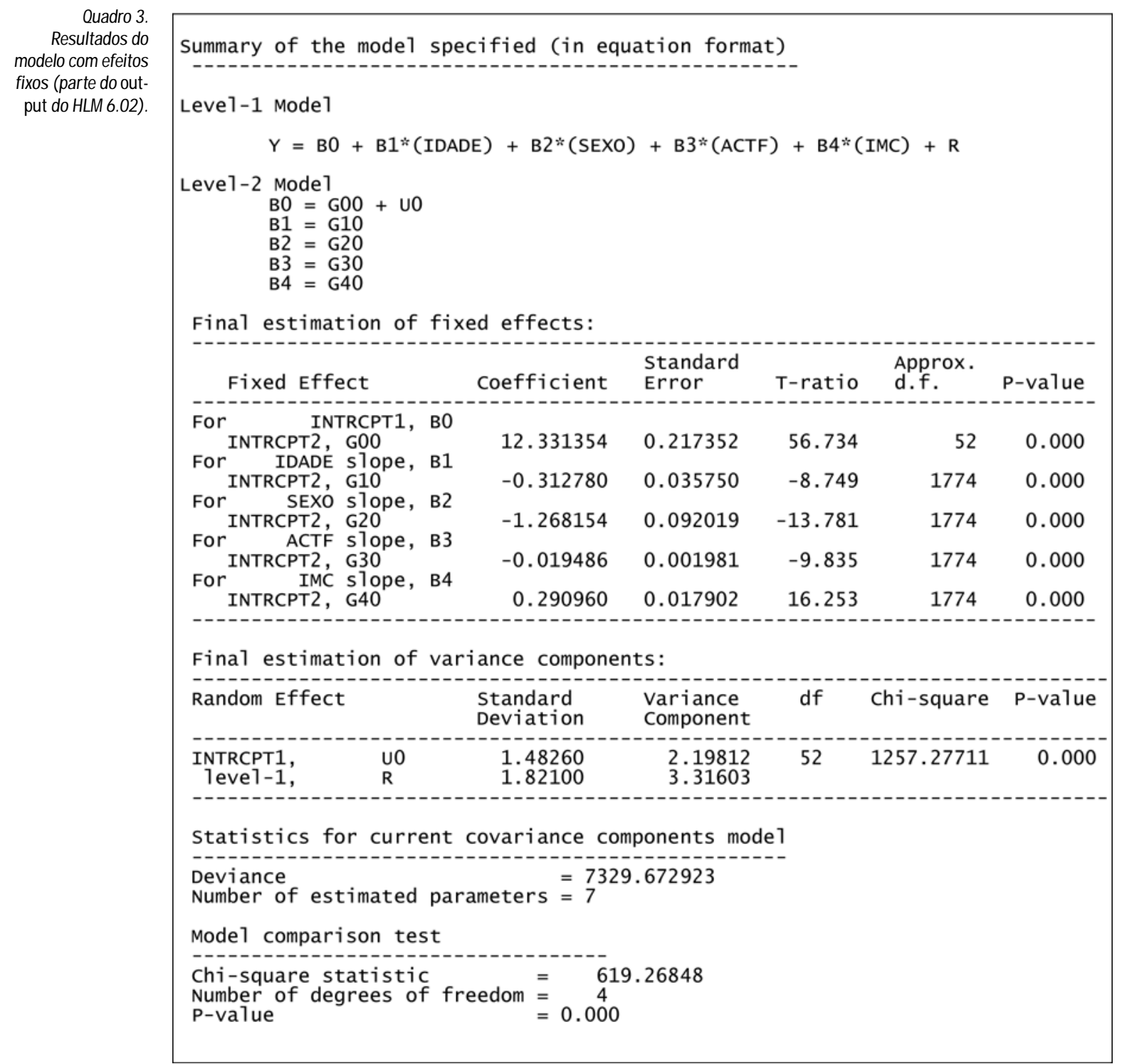

Interpretemos o output nos seus diferentes aspectos: Primeiro: A grande média do desempenho al terouse pela circunstância de haver agora predictores no modelo. 0 seu valor é de 12,3 minutos. O que é que este valor significa? É o valor médio correspondente ao desempenho das meninas, uma vez que a variável referência relativa ao sexo no modelo é zero para as meninas, e um para os meninos.

Segundo: $O$ aumento da idade implica uma redução significativa do tempo de prova, em cerca de 0,31 minutos por ano de idade $(B 1=-0,31 \pm 0,05$ minutos). Os meninos têm performances superiores às meninas, em média uma redução significativa no tempo de desempenho de 1,27 minutos ( $B 2=$ $1,27 \pm 0,14)$. As crianças mais activas são as que percorrem a distância em menos tempo (B3= $0,02 \pm 0,005$ ). As que têm valores mais elevados de IMC são penalizadas na prova, em média 0,29 minutos (B4=0,29 $\pm 0,02)$.

Terceiro: Ainda há variância inter-individual por 

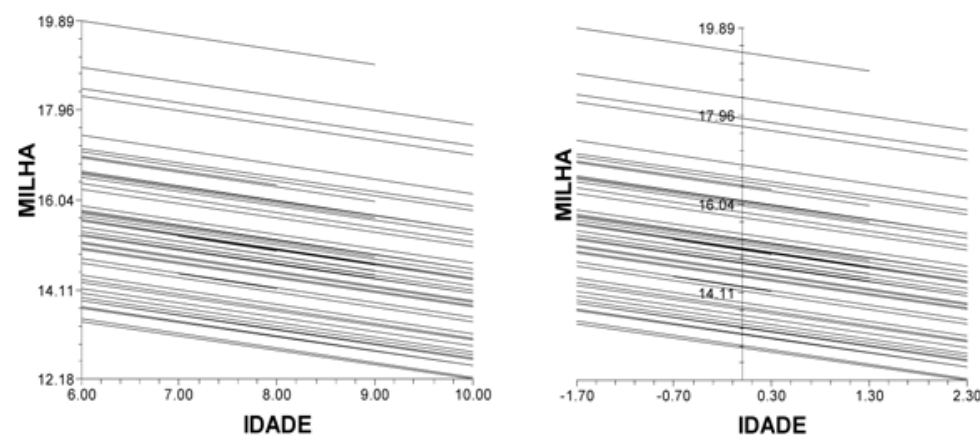

Figura 2. Trajectórias modeladas do desempenho dos sujeitos das diferentes escolas no modelo de efeitos fixos (gráficos obtidos no HLM 6.02)

Sexto: Qual é a variância explicada, ao nível do desempenho dos alunos, pela entrada destes predictores? A resposta é bem simples. Basta para tanto contrastar as variâncias residuais entre os dois modelos, de tal modo que a variância explica$\mathrm{da}=(4,74675-$ 3,31603)/4,74675=0,30. O conjunto dos predictores idade, sexo, ActF e IMC explica $30 \%$ da variância total do desempenho interindividual, o que representa um val or elevado.

Na Figura 2 encontram-se as trajectórias dos sujeitos de todas as escolas.

explicar, e cujo valor é de 3,32. Está aberto o espaço para a inclusão de outros predictores que não foram pensados nesta pesquisa e que futuros investigadores deverão considerar, como aspectos da economia de corrida, percepção do tempo de prova, motivação para a sua realização, amplitude da passada que depende do comprimento do membro inferior, etc. Quarto: Há variância significativa no desempenho médio entre escolas, cuja magnitude é de 2,20, que é estatisticamente significativa ( $p<0,001)$, e que mais adiante modelaremos.

Q uinto: Dado o esforço de modelação, é importante saber da sua qualidade. Isto é, precisamos não só de informação acerca do significado estatístico dos parâmetros incluídos (essa informação é providenciada pelo output), bem como de uma estatística que nos informe acerca do valor de cada modelo. Essa estatística é designada de $D$ eviance. $A$ D eviance só faz sentido quando comparada com modelos contidos em si mesmos (do inglês nested within). Ora relativamente ao modelo nulo, este que agora elaboramos, o de efeitos fixos, é mais expansivo, e neste sentido pode ser comparado com o modelo nulo. Assim, a D eviance de cada modelo é sempre apresentada no final do output, bem como do número de parâmetros estimados. No HLM 6.02 há uma opção de teste de hipóteses, que permite comparar dois modelos entre si desde que sejam nested within, isto é, hierarquicamente contidos. O resultado encontra-se numa parte designada de model comparison test. Dado que o resultado é estatisticamente significativo $\left(c^{2}{ }_{(4)}=619,27, p<0,001\right)$, o nosso esforço de modelação foi bem sucedido.
Chamamos a atenção dos leitores para as diferenças nos dois gráficos, que reclamam duas explicações para serem entendidos:

como se assumiu que o modelo era de trajectórias fixas, todas as rectas de regressão relativas as desempenho são paralelas cujo declive é negativo. Isto significa que com o aumento da idade o tempo para percorrer a distância é cada vez menor.

A idade (na abcissa) está expressa em duas métricas distintas*. Na esquerda nos val ores originais da idade dos sujeitos. Esta métrica coloca al guma dificuldade de interpretação, dado que, conceptualmente, o valor na ordenada corresponde ao desempenho das crianças quando a sua idade é zero, o que é uma impossibilidade (não obstante estar nos seis anos de idade). O valor zero não tem pois qualquer significado, uma vez que à idade zero não pode corresponder qualquer desempenho na prova. Na figura da direita os diferentes valores de idade de todos os sujeitos foram subtraídos à média global. O que aqui temos são, pois, dados centrados e logicamente interpretáveis, dado que o val or médio global do desempenho (o valor na ordenada) corresponde agora a uma criança média de idade igual a 7,69 anos, o que tem todo o sentido. Não é pois de estra nhar os valores de idade que aparecem na abcissa (a idade de 7,69 corresponde ao zero da abcissa). O valor na ordenada (intercept) corresponde ao desempenho de uma criança cujo valor é igual à média da sua respectiva escola. Na situação deste modelo, as rectas são paral elas por não se ter assumido que diferenças nas distribuições de idades, sexos e valores do IMC distinguiria o desempenho entre escolas. 


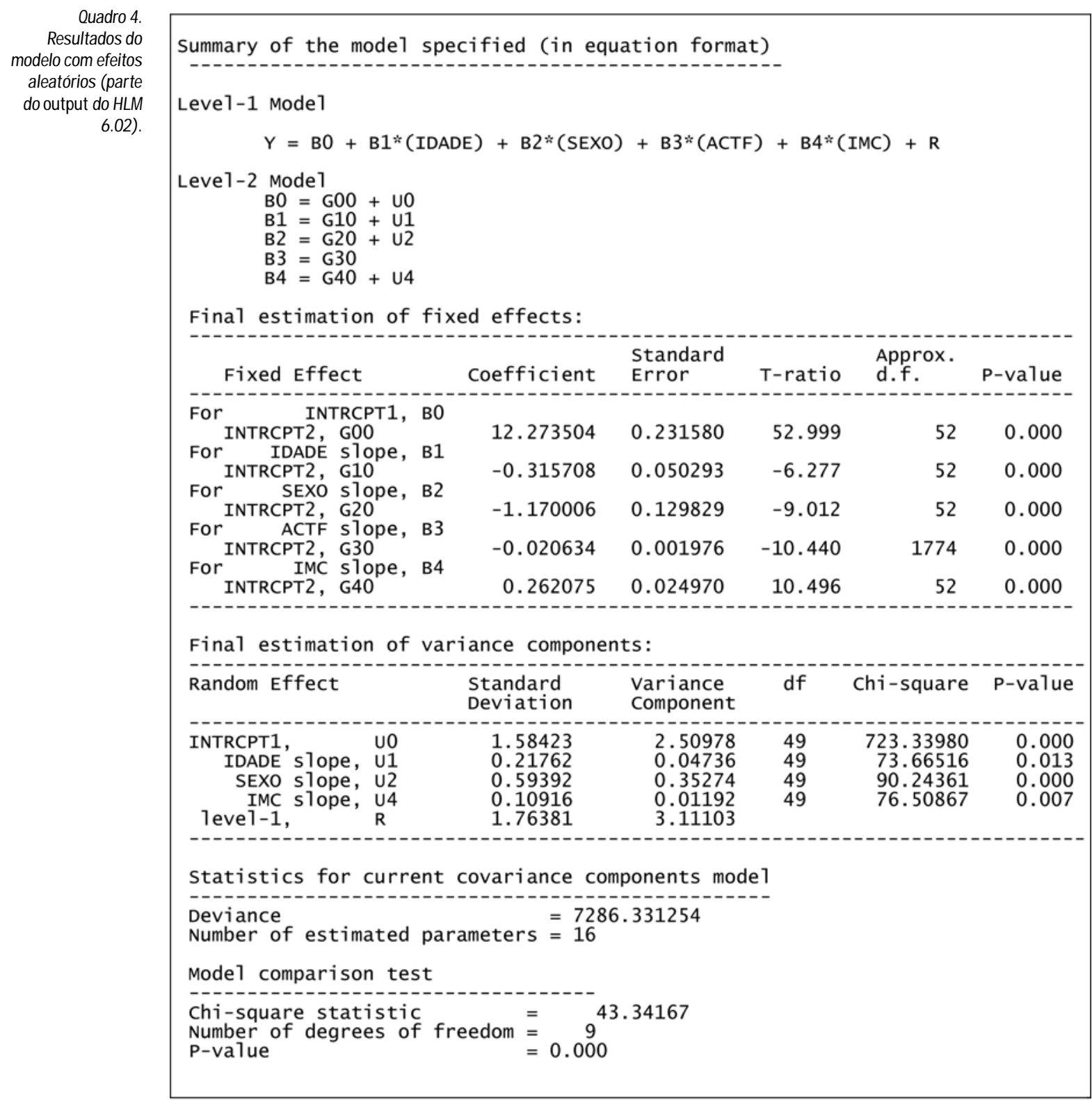

Etapa no 3: Modelo de efeitos aleatórios (do inglês Level1 random-intercepts and random-slopes model) Tornando um pouco mais complexa a análise, e no sentido de explorar ainda mais o conteúdo da informação disponível, poderia pensar-se que os desempenhos entre escolas sejam função da distribuição da idade das crianças (e nem todas têm o mesmo efectivo e a mesma distribuição de idades), das diferenças nos efectivos de crianças de ambos os sexos na composição das turmas, e nas diferenças entre valores do IMC. Trata-se, agora de modelar aspectos aleatórios destas variáveis no contexto das suas respectivas escolas. Os resultados estão no Quadro 4, e na Figura 3, onde são bem marcantes as diferenças nos dedives entre escolas, dado que o seu desempenho é condicionado pelas variáveis referidas no parágrafo anterior. 


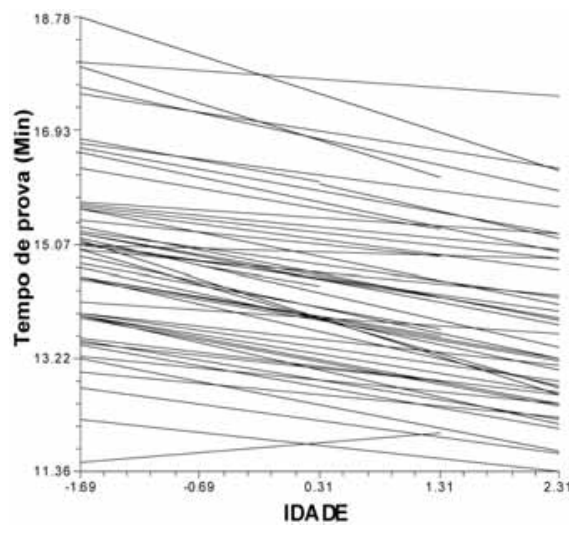

Figura 3. Trajectórias modeladas do desempenho dos sujeitos das diferentes escolas, no modelo de efeitos aleatórios ( gráfico obtido no HLM 6.02)

Interpretemos, então, os resultados do Quadro 4, tendo sempre presente que há diferenças nos valores relativamente ao modelo anterior dadas as novas condições deste modelo:

Primeiro: A grande média do desempenho é agora de 12,27 minutos, cuja interpretação nos é agora familiar. Segundo: O aumento da idade implica uma redução significativa do tempo de prova em cerca de 0,32 minutos ( $B 1=-0,32 \pm 0,05$ minutos). Os meninos têm performances superiores às meninas, em média uma redução significativa no desempenho de 1,17 minutos ( $B 2=-1,7 \pm 0,13)$. Os mais activos são os que cobrem a distância em menos tempo (B3=$0,02 \pm 0,002)$. Os que têm val ores mais elevados de IMC são penal izados na prova, em média 0,26 minutos (B4=0,26 $\pm 0,02$ ).

Terceiro: Ainda há variância inter-individual por explicar, e cujo valor é de 3,11. As sugestões anteriormente apresentadas são também transferíveis para aqui.

Quarto: Há variância significativa no desempenho médio entre escolas, cuja magnitude é de 2,50 que é estatisticamente significativa $(p<0,001)$.

Quinto: Ficou evidente que as variáveis idade, sexo e IMC são significativas na parte aleatória do modelo, sugerindo que a variação da sua distribuição pelas escolas implica uma atenção adequada para se entender o seu significado.
Sexto: A D eviance deste modelo passou a ser de 7286,33 , que contrastada com a do modelo anterior se revel ou estatisticamente significativa $\left(\chi^{2}{ }_{(9)}=43,34, p<0,001\right)$, implicando o sucesso do nosso esforço de modelação.

Sétimo: Qual é a variância explicada, ao nível do desempenho dos al unos, por este modelo? A resposta é bem simples. Basta para tanto contrastar as variâncias residuais entre os dois modelos, o nulo e este, tal que a variância explicada $=(4,74675$ $3,11103) / 4,74675=0,35$. Relativamente ao modelo com efeitos fixos, ganhou-se cerca de $5 \%$.

Etapa nㄴ: Modelo com predictores do 2ำ nível (do inglês intercepts and slopes as outcomes) Resta agora a tarefa de maior dificuldade e que implica algum esforço de contenção no uso dos predictores entre escolas. Da lista apresentada no Quadro 1, dos oito predictores, temos al guma dificuldade na sua escolha por dois motivos fundamentais: não conhecemos qual quer esforço de teorização relativamente ao desempenho motor de crianças deste nível de ensino, qualquer que seja a estrutura operativa da bateria de testes utilizada.

A mai or parte dos predictores têm uma variabilidade extremamente reduzida entre escolas. Daqui que a nossa escolha recaia sobre a variabilidade da localização das escolas (variável MEIO), e na diversidade material e de espaço do recreio escolar (RECREIO). Os resultados deste nosso esforço estão no Quadro 5. A sua interpretação está condicionada ao valor da D eviance e à comparação correspondente com o modelo anterior. Ora o resultado do valor de prova $(p=0,35)$ e que está na última linha do output, implica que o aumento de complexidade deste modelo não redundou em aumento de capacidade explicativa. N esta situação preferimos um modelo mais parcimonioso, precisamente $o$ anterior (Level-1 randomintercept and random-slopes model). Dito de um modo mais explícito, este modelo, enquanto intérprete da complexidade organizacional do desempenho situado em ambos os níveis da hierarquia, não se ajusta bem aos dados disponíveis.

Tentemos interpretar o porquê desta "fal ha" do modelo apresentado pelos autores para interpretar os resultados na prova da milha, e não o modelo estatístico subjacente à MHMN. Do ponto de vista 
Quadro 5. Resultados do modelo com predictores ao nível do envolvimento ( parte do output do HLM 6.02).

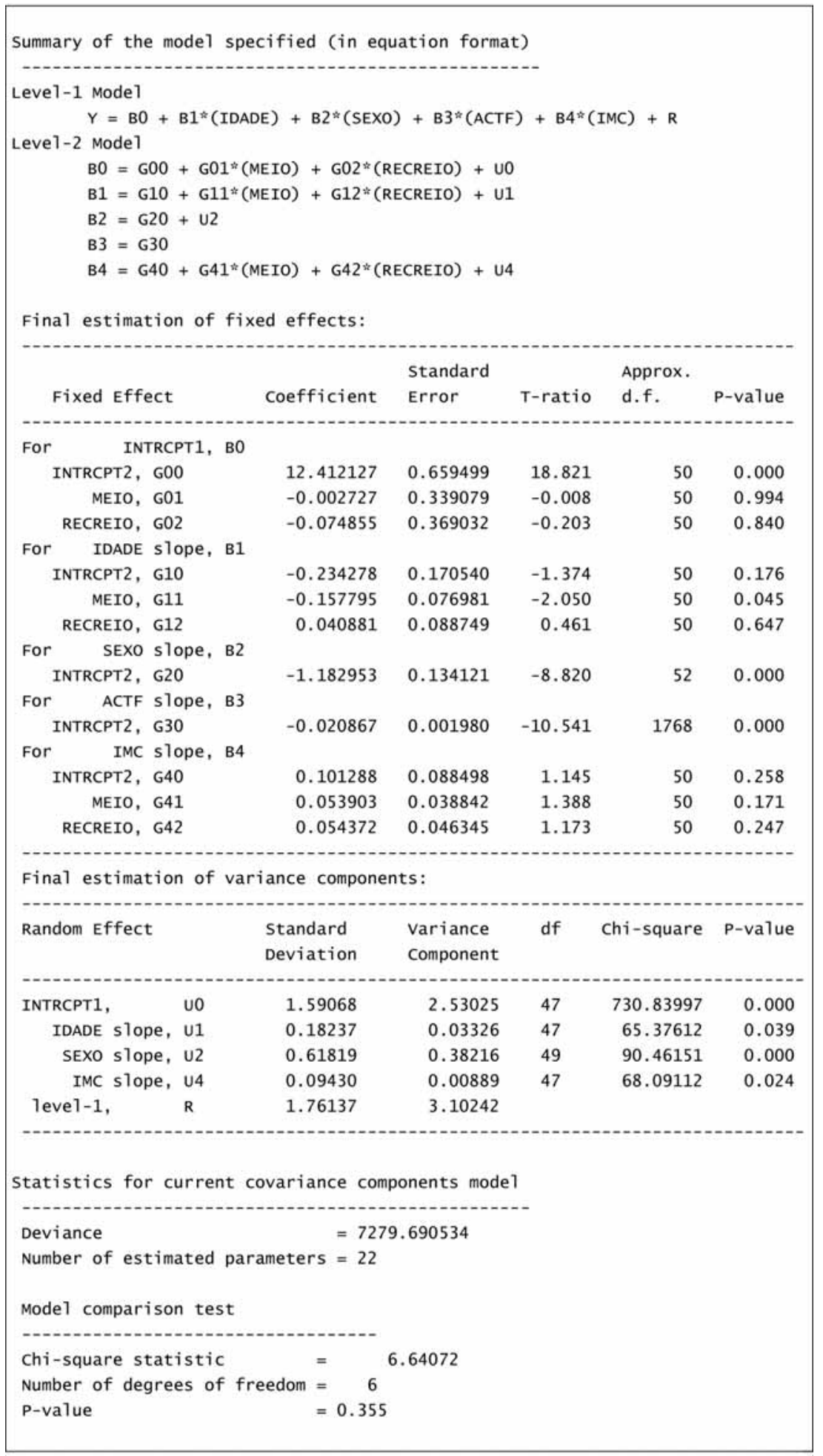


"ecológico" faria todo o sentido em atribuir significado às condições do ambiente (meio sócio-económico de implantação da escola, condições materiais e espaciais dos recreios) para dar algum significado às diferenças de desempenho entre crianças pertencentes a essas escolas. Tal não aconteceu. Contudo, não nos parece que condições externas às crianças não condicionem o seu desempenho na prova da milha, um indicador indirecto da sua resistência aeróbia, dado que nenhum dos parâmetros estimados para tais variáveis seja estatisticamente significativo. A literatura da especialidade (concretamente a que utiliza delineamentos gemelares ou de famílias nucleares) bem sumariada no texto de Bouchard et al. (5) sugere que os factores genéticos explicam aproximadamente cerca de $50 \%$ da variação total da resistência aeróbia. Há pois um espaço de outros $50 \%$ de variabilidade que deve ser encontrado nas forças vivas e sempre cambiantes do meio envolvente, cuja influência no desempenho e resposta ao treino são importantes.

Pode ter bem acontecido que a operacionalização dos factores do envolvimento considerados neste estudo não tenha sido a mais adequada. Neste sentido, chamamos a atenção dos leitores para a necessidade de um maior cuidado na operacionalização das condicionantes ambientais, sobretudo na construção da base de dados de indicadores que com maior validade de conteúdo reflictam estas variáveis. Este é um exemplo "real" recheado dos ingredientes de al guma incerteza que povoam a investigação empírica. A sua exploração é sempre bem diferente e mais contingencial da que ocorre com os exemplos construídos para serem incluídos em manuais de MHMN. Daqui a sua vantagem e valor ao permitir uma discussão mais esclarecedora dos resultados e dos problemas que estão normalmente associados à operacionação das variáveis, i.e., a aspectos da sua validade. Finalmente para salientar, que no estudo de Zhu(30) e precisamente na prova da milha, os factores do envolvimento mais relevantes eram a presença de um professor de EF qualificado a dar as aulas às crianças, bem como a avaliação da sua AptF e a interpretação, juntamente com todos os alunos de cada classe, do significado dos resultados obtidos e o estabelecimento de objectivos adequados para promover uma melhor AptF. Ora no estudo de Sousa e
Maia(27) das 53 escolas amostradas, somente uma tinha aulas com um professor de EF. Um triste facto para todas as crianças.

\section{CONSIDERAÇÕES FINAIS}

Em resumo, quando se considera informação relativa ao desempenho motor de crianças e jovens, e cujos dados estejam constituídos por uma estrutura organizacional (e esta é a maior parte das situações), é importante recorrer a MHMN para extrair todo "a essência" contida nos resultados. A não consideração desta condição implica um viés sério em termos de interpretação do desempenho. A riqueza da análise e da atribuição de significado aos parâmetros estimados por este tipo de metodologias representa um salto qualitativo enorme na exploração do desempenho, e sobretudo no estabel ecimento de hipóteses mais abrangentes e esclarecedoras.

\section{AGRADECIMENTOS}

Os autores agradecem ao revisor anónimo os comentários que permitiram clarear diferentes aspectos do trabalho. Do mesmo modo agradecem à Fundação para a Ciência e Tecnologia o apoio na realização deste estudo (POCI/DES/62499/2004).

\section{CORRESPONDÊNCIA}

\section{José António Ribeiro Maia}

Faculdade de Desporto

Universidade do Porto

Rua Dr. Plácido Costa, 91

4200-450 Porto

Portugal

E-mail: jmaia@fade.up.pt 


\section{REFERÊNCIAS}

1. Almeida CMPS, Marques AT, Maia JAR (2001) A ptidão física, estatuto sócio-económico, e medidas antropométricas da população escolar do concelho de Lamego. Estudo em crianças e jovens de ambos os sexos dos 10 aos 16 anos de idade. Lamego: Câmara Municipal de Lamego.

2. Baxter-Jones A, Goldstein H, Helms P (1993) The development of aerobic power in young athletes. J A ppl Physiol 75:1160-1167.

3. Beunen G, Baxter-Jones ADG, Mirwald RL, Thomis M, Lefevre J, Malina RM, Bailey DA (2002). Intraindividual allometric development of aerobic power in 8- to 16-yearold boys. M ed Sci Sports Exerc 33: 503-510.

4. Boyle MH, Willms JD (2001). Multilevel modelling of hierarchical data in developmental studies. J Child Psychol Psychiat 42: 141-162.

5. Bouchard C, Malina RM, Pérusse L (1997). Genetics of fitness and physical performance. Champaign: Human Kinetics Publishers.

6. Campbell SK, Hedecker D (2001). Validity of the test of infant motor performance for discriminating among infants with varying risk for poor motor outcome. J Pediatr 139: 546-551.

7. Courgeau D (2003). M ethodology and epistemology of multilevel analysis. A pproaches from different social sciences. Dordrecht: Kluwer Academic Press.

8. Duncan C, Jones K, Moon G (1998). Context, Composition and heterogeneity: using multilevel models in health research. Soc Sci M ed 46: 97-117.

9. Ferreira JCV, Marques AT, Maia JR (2002). A ptidão física, actividade física e saúde da população escolar do centro da área educativa de Viseu. U m estudo em crianças e jovens de ambos os sexos dos 10 aos 18 anos de idade. Viseu: Instituto Politécnico de Viseu.

10. Freitas DL, Maia JA, Beunen GP, Lefevre JA, Claessens AL, Marques AT, Rodrigues AL, Silva CA, Crespo MT (2002). Crescimento somático, maturação biológica, aptidão física e estatuto sócio-económico de crianças e adolescentes madeirenses. 0 estudo de crescimento da M adeira. Funchal: Universidade da Madeira.

11. Godin G, Shephard RJ (1985). A simple method to assess exercise behaviour in the community. Can J A ppl Sport Sci 10:141-146.

12. Goldstein H, Rasbash J, Yang M, Woodhouse G (1993). A multilevel analysis of school examination results. 0 xford Review of Education 19:425-433.

13. Guedes DP, Guedes JE (1997). Crescimento, composição corporal e desempenho motor de crianças e adolescentes. Ed. Baliero. São Paulo.

14. Heck RH, Thomas SL (2000). A n introduction to multilevel modeling techniques. Mahwah: Lawrence Erlbaum Associates.

15. Hox J (2002). Multilevel analysis. Techniques and applications. Mahwah: Lawrence Erlbaum Associates.
16. Maia JAR, Lopes VP, Silva RG, Seabra A, Ferreira JV, Cardoso MV (2003). Modelação hierárquica ou multinível. Uma metodologia estatística e um instrumento útil de pensamento na investigação em Ciências do Desporto. Rev Port Cien Desp 3: 92-107.

17. Maia JAR, Lopes VP, Morais FP, Silva RMG, Seabra A (2002). Estudo do crescimento somático, aptidão física, actividade física e capacidade de coordenação corporal de crianças do 1 으 ciclo do ensino básico da Região A utónoma dos A çores. Porto e Ponta Delgada: DREFD, FCDEF-UP.

18. Marcondes, E. (1982) Crescimento e desenvolvimento pubertário em crianças e adolescentes brasileiros II - altura e peso. São Paulo. Editora Brasileira de Ciências.

19. Matsudo, VKR (1992). Critérios biológicos para diagnóstico, prescrição de aptidão física em escolares de 7 aos 18 anos de idade. Tese de Livre-Docência. Rio de Janeiro, Universidade Gama Filho.

20. Nahas MV, Petroski EL, Jesus JF, Silva OJ (1992) Crescimento e aptidão física relacionada à saúde em escolares de 7 a 10 anos - Um estudo longitudinal. Rev Bras Cien Esporte 14: 7-17.

21. Nevill AM, Holder RL (2000). Modelling health-related performance indices. A nn Hum Biol 27: 543-559.

22. Papaioannou A, Marsh HW, Theodorakis Y (2004). A multilevel approach to motivational climate in physical education and support setting: an individual or a group level construct? J Sport Exerc Psychology 26: 90-118.

23. Raudenbush SW, Bhumirat C (1992) The distribution of resources for primary education and its consequences for education achievement in Thailand. Int J Edu Res 143-164.

24. Raudenbush SW, Bryk A (2002). H ierarchical linear models. A pplications and data analysis methods. 2nd edition. Thousand Oaks: Sage Publications, Inc.

25. Sastry N, Ghosh-Dastidar B, Adams J, Pebley AR (2003). The design of a multilevel survey of children, families, and communities: the Los A ngeles family and neighbourhood survey. Santa Mónica: RAND Corporation.

26. Schreiber JB, Griffin BW (2004). Review of multilevel modelling and multilevel studies in the Journal of Education Research (1992-2002). J Edu Res 1: 24-33.

27. Sousa MA, Maia JAR (2005). Crescimento somático, actividade física e aptidão física associada à saúde. Um estudo populacional nas crianças do 1 o ciclo do ensino básico do concel ho de A marante. Edições da Câmara Municipal de Amarante e FCDEF-UP. Amarante e Porto.

28. Tinklin T (2000). The influence of social background on application and entry to higher education in Scotland: a multilevel analysis. Higher Edu Q uart 54: 343-385.

29. Waltrick AC, Duarte MF (2000). Estudo das características antropométricas de escolares de 7 a 17 anos - Uma abordagem longitudinal mista e transversal. R ev Bras Cine D es H um 2: 17-30.

30. Zhu W (1997). A multilvel analysis of school factors associated with health-related fitness. R es Q uart Exerc Sport 68:125-135. 